\title{
Free Field Approach to Solutions of the Quantum Knizhnik-Zamolodchikov Equations
}

\author{
Kazunori KUROKI ${ }^{\dagger}$ and Atsushi NAKAYASHIKI ${ }^{\ddagger}$ \\ $\dagger$ Department of Mathematics, Kyushu University, Hakozaki 6-10-1, Fukuoka 812-8581, Japan \\ E-mail: ma306012@math.kyushu-u.ac.jp \\ $\ddagger$ Department of Mathematics, Kyushu University, Ropponmatsu 4-2-1, Fukuoka 810-8560, Japan \\ E-mail:6vertex@math.kyushu-u.ac.jp
}

Received February 18, 2008, in final form May 27, 2008; Published online June 03, 2008

Original article is available at http://www.emis.de/journals/SIGMA/2008/049/

\begin{abstract}
Solutions of the qKZ equation associated with the quantum affine algebra $U_{q}\left(\widehat{s l}_{2}\right)$ and its two dimensional evaluation representation are studied. The integral formulae derived from the free field realization of intertwining operators of $q$-Wakimoto modules are shown to coincide with those of Tarasov and Varchenko.
\end{abstract}

Key words: free field; vertex operator; $\mathrm{qKZ}$ equation; $q$-Wakimoto module

2000 Mathematics Subject Classification: 81R50; 20G42; 17B69

\section{Introduction}

In 1992 I. Frenkel and N. Reshetikhin [7] had developed the theory of intertwining operators for quantum affine algebras and had shown that the matrix elements of intertwiners satisfy the quantized Knizhnik-Zamolodchikov (qKZ) equations.

The theory of intertwiners and qKZ equations was successfully applied to the study of solvable lattice models [9] (and references therein). As to the study of solutions of the qKZ equations, bases are constructed by Tarasov and Varchenko [22] in the form of multi-dimensional hypergeometric integrals in the case of $U_{q}\left(\widehat{s l}_{2}\right)$. However solutions of the qKZ equations for other quantum affine algebras are not well studied [21].

The method of free fields is effective to compute correlation functions in conformal field theory (CFT) [3], in particular, solutions to the Knizhnik-Zamolodchikov (KZ) equations [18, 19]. A similar role is expected for those of quantum affine algebras. Unfortunately it is difficult to say that this expectation is well realized, as we shall explain below.

Free field realizations of quantum affine algebras are constructed by Frenkel and Jing [6] for level one integrable representations of ADE type algebras and by Matsuo [15], Shiraishi [16] and Abada et al. [1] for representations with arbitrary level of $U_{q}\left(\widehat{s l}_{2}\right)$. The latter results are extended to $U_{q}\left(\widehat{s l}_{N}\right)$ in [2]. Free field realizations of intertwiners are constructed based on these representations in the case of $U_{q}\left(\widehat{s l}_{2}\right)[9,10,12,15,4]$.

The simplicity of the Frenkel-Jing realizations makes it possible not only to compute matrix elements but also traces of intertwining operators [9], which are special solutions to the qKZ equations. The case of $q$-Wakimoto modules with an arbitrary level becomes more complex and the detailed study of the solutions of the qKZ equations making use of it is not well developed. In [15] Matsuo derived his integral formulae [14] from the formulae obtained by the free field calculation in the simplest case of one integration variable. However it is not known in general whether the integral formulae derived from the free field realizations recover those of $[14,23,22]^{1}$.

\footnotetext{
${ }^{1}$ See Note 1 in the end of the paper.
} 
The aim of this paper is to study this problem in the case of the qKZ equation with the value in the tensor product of two dimensional irreducible representations of $U_{q}\left(\widehat{s l}_{2}\right)$. More general cases will be studied in a subsequent paper.

There are mainly two reasons why the comparison of two formulae is difficult. One is that the formulae derived from the free field calculations contain more integration variables than in Tarasov-Varchenko's (TV) formulae. This means that one has to carry out some integrals explicitly to compare two formulae. The second reason is that the formulae from free fields contains a certain sum. This stems from the fact that the current and screening operators are written as a sum which is absent in the non-quantum case. Since TV formulae have a similar structure to those for the solutions of the $\mathrm{KZ}$ equation $[18,19]$, one needs to sum up certain terms explicitly for the comparison of two formulae. We carry out such calculations in the case we mentioned.

The plan of this paper is as follows. In Section 2 the construction of the hypergeometric solutions of the qKZ equation due to Tarasov and Varchenko is reviewed. The free field construction of intertwining operators is reviewed in Section 3. In Section 4 the formulae for the highest to highest matrix elements of some operators are calculated. The main theorem is also stated in this section. The transformation of the formulae from free fields to Tarasov-Varchenko's formulae is described in Section 5. In Section 6 the proof of the main theorem is given. Remaining problems are discussed in Section 7. The appendix contains the list of the operator product expansions which is necessary to derive the integral formula.

\section{Tarasov-Varchenko's formula}

Let $V^{(1)}=\mathbf{C} v_{0} \oplus \mathbf{C} v_{1}$ be a two-dimensional irreducible representation of the algebra $U_{q}\left(s l_{2}\right)$, and $R(z) \in \operatorname{End}\left(V^{(1) \otimes 2}\right)$ be a trigonometric quantum $R$-matrix given by

$$
\begin{aligned}
& R(z)\left(v_{\epsilon} \otimes v_{\epsilon}\right)=v_{\epsilon} \otimes v_{\epsilon}, \\
& R(z)\left(v_{0} \otimes v_{1}\right)=\frac{1-z}{1-q^{2} z} q v_{0} \otimes v_{1}+\frac{1-q^{2}}{1-q^{2} z} v_{1} \otimes v_{0}, \\
& R(z)\left(v_{1} \otimes v_{0}\right)=\frac{1-q^{2}}{1-q^{2} z} z v_{0} \otimes v_{1}+\frac{1-z}{1-q^{2} z} q v_{1} \otimes v_{0},
\end{aligned}
$$

Let $p$ be a complex number such that $|p|<1$ and $T_{j}$ denote the multiplicative $p$-shift operator of $z_{j}$,

$$
T_{j} f\left(z_{1}, \ldots, z_{n}\right)=f\left(z_{1}, \ldots, p z_{j}, \ldots, z_{n}\right) .
$$

The qKZ equation for the $V^{(1) \otimes n}$-valued function $\Psi\left(z_{1}, \ldots, z_{n}\right)$ is

$$
T_{j} \Psi=R_{j, j-1}\left(p z_{j} / z_{j-1}\right) \cdots R_{j, 1}\left(p z_{j} / z_{1}\right) \kappa^{\frac{1-h_{j}}{2}} R_{j, n}\left(z_{j} / z_{n}\right) \cdots R_{j, j+1}\left(z_{j} / z_{j+1}\right) \Psi,
$$

where $R_{i j}(z)$ signifies that $R(z)$ acts on the $i$-th and $j$-th components, $\kappa$ is a complex parameter, $\kappa^{\frac{1-h_{j}}{2}}$ acts on the $j$-th component as

$$
\kappa^{\frac{1-h_{j}}{2}} v_{\epsilon}=\kappa^{\epsilon} v_{\epsilon}
$$

Let us briefly recall the construction of the hypergeometric solutions [22, 20] of the equation (1). In the remaining part of the paper we assume $|q|<1$. We set

$$
(z)_{\infty}=(z ; p)_{\infty}, \quad(z ; p)_{\infty}=\prod_{j=0}^{\infty}\left(1-p^{j} z\right), \quad \theta(z)=(z)_{\infty}\left(p z^{-1}\right)_{\infty}(p)_{\infty}
$$


Let $n$ and $l$ be non-negative integers satisfying $l \leq n$. For a sequence $(\epsilon)=\left(\epsilon_{1}, \ldots, \epsilon_{n}\right) \in$ $\{0,1\}^{n}$ satisfying $\sharp\left\{i \mid \epsilon_{i}=1\right\}=l$ let

$$
w_{(\epsilon)}(t, z)=\prod_{a<b} \frac{t_{a}-t_{b}}{q^{-2} t_{a}-t_{b}} \sum_{\substack{1 \leq a_{1}, \ldots, a_{l} \leq l \\ a_{i} \neq a_{j}(i \neq j)}} \prod_{i=1}^{l}\left(\frac{t_{a_{i}}}{t_{a_{i}}-q^{-1} z_{k_{i}}} \prod_{j<k_{i}} \frac{q^{-1} t_{a_{i}}-z_{j}}{t_{a_{i}}-q^{-1} z_{j}} \prod_{i<j} \frac{q^{-2} t_{a_{i}}-t_{a_{j}}}{t_{a_{i}}-t_{a_{j}}}\right),
$$

where $\left\{i \mid \epsilon_{i}=1\right\}=\left\{k_{1}<\cdots<k_{l}\right\}$.

The elliptic hypergeometric space $\mathcal{F}_{\text {ell }}$ is the space of functions $W(t, z)=W\left(t_{1}, \ldots, t_{l}\right.$, $\left.z_{1}, \ldots, z_{n}\right)$ of the form

$$
W=Y(z) \Theta(t, z) \frac{1}{\prod_{j=1}^{n} \prod_{a=1}^{l} \theta\left(q t_{a} / z_{j}\right)} \prod_{1 \leq a<b \leq l} \frac{\theta\left(t_{a} / t_{b}\right)}{\theta\left(q^{-2} t_{a} / t_{b}\right)}
$$

satisfying the following conditions

(i) $Y(z)$ is meromorphic on $\left(\mathbb{C}^{*}\right)^{n}$ in $z_{1}, \ldots, z_{n}$, where $\mathbb{C}^{*}=\mathbb{C} \backslash\{0\}$;

(ii) $\Theta(t, z)$ is holomorphic on $\left(\mathbb{C}^{*}\right)^{n+l}$ in $t_{1}, \ldots, z_{n}$ and symmetric in $t_{1}, \ldots, t_{l}$;

(iii) $T_{a}^{t} W / W=\kappa q^{n-2 l+4 a-2}, T_{j}^{z} W / W=q^{-l}$, where $T_{a}^{t} W=W\left(t_{1}, \ldots, p t_{a}, \ldots t_{l}, z\right)$ and $T_{j}^{z} W=$ $W\left(t, z_{1}, \ldots, p z_{j}, \ldots z_{n}\right)$.

Define the phase function $\Phi(t, z)$ by

$$
\Phi(t, z)=\prod_{i=1}^{n} \prod_{a=1}^{l} \frac{\left(q t_{a} / z_{i}\right)_{\infty}}{\left(q^{-1} t_{a} / z_{i}\right)_{\infty}} \prod_{a<b} \frac{\left(q^{-2} t_{a} / t_{b}\right)_{\infty}}{\left(q^{2} t_{a} / t_{b}\right)_{\infty}} .
$$

For $W \in \mathcal{F}_{\text {ell }}$ let

$$
I\left(w_{(\epsilon)}, W\right)=\int_{\tilde{\mathbb{T}}^{l}} \prod_{a=1}^{l} \frac{d t_{a}}{t_{a}} \Phi(t, z) w_{(\epsilon)}(t, z) W(t, z),
$$

where $\tilde{\mathbb{T}}^{l}$ is a suitable deformation of the torus

$$
\mathbb{T}^{l}=\left\{\left(t_{1}, \ldots, t_{l}\right)|| t_{i} \mid=1,1 \leq i \leq l\right\},
$$

specified as follows [22].

Notice that the integrand has simple poles at

$$
\begin{aligned}
& t_{a} / z_{j}=\left(p^{s} q^{-1}\right)^{ \pm 1}, \quad s \geq 0, \quad 1 \leq a \leq l, \quad 1 \leq j \leq n, \\
& t_{a} / t_{b}=\left(p^{s} q^{2}\right)^{ \pm 1}, \quad s \geq 0, \quad 1 \leq a<b \leq l .
\end{aligned}
$$

The contour for the integration variable $t_{a}$ is a simple closed curve which rounds the origin in the counterclockwise direction and separates the following two sets,

$$
\begin{aligned}
& \left\{p^{s} q^{-1} z_{j}, p^{s} q^{2} t_{b} \mid s \geq 0,1 \leq j \leq n, a<b\right\}, \\
& \left\{p^{-s} q z_{j}, p^{-s} q^{-2} t_{b} \mid s \geq 0,1 \leq j \leq n, a<b\right\} .
\end{aligned}
$$

Then

$$
\Psi_{W}=\sum_{(\epsilon)} I\left(w_{(\epsilon)}, W\right) v_{\epsilon_{1}} \otimes \cdots \otimes v_{\epsilon_{n}},
$$

is a solution of the $\mathrm{qKZ}$ equation (1) for any $W \in \mathcal{F}_{\text {ell }}$. 


\section{Free field realizations}

In this section we review the free field construction of the representation of the quantum affine algebra $U_{q}\left(\widehat{s l_{2}}\right)$ of level $k$ and intertwining operators. We mainly follow the notation in [10]. We set

$$
[x]=\frac{q^{x}-q^{-x}}{q-q^{-1}} .
$$

Let $k$ be a complex number and $\left\{a_{n}, b_{n}, c_{n}, \tilde{a}_{0}, \tilde{b}_{0}, \tilde{c}_{0}, Q_{a}, Q_{b}, Q_{c} \mid n \in \mathbb{Z} \backslash\{0\}\right\}$ satisfy

$$
\begin{array}{rlrl}
{\left[a_{n}, a_{m}\right]} & =\delta_{m+n, 0} \frac{[2 n][(k+2) n]}{n}, & & {\left[\tilde{a}_{0}, Q_{a}\right]=2(k+2),} \\
{\left[b_{n}, b_{m}\right]=-\delta_{m+n, 0} \frac{[2 n]^{2}}{n},} & {\left[\tilde{b}_{0}, Q_{b}\right]=-4,} \\
{\left[c_{n}, c_{m}\right]=\delta_{m+n, 0} \frac{[2 n]^{2}}{n},} & {\left[\tilde{c}_{0}, Q_{c}\right]=4,}
\end{array}
$$

Other combinations of elements are supposed to commute. Set

$$
N_{ \pm}=\mathbb{C}\left[a_{n}, b_{n}, c_{n} \mid \pm n>0\right] .
$$

Then the Fock module $F_{r, s}$ is defined to be the free $N_{-}$-module of rank one generated by the vector which satisfies

$$
N_{+}|r, s\rangle=0, \quad \tilde{a}_{0}|r, s\rangle=r|r, s\rangle, \quad \tilde{b}_{0}|r, s\rangle=-2 s|r, s\rangle, \quad \tilde{c}_{0}|r, s\rangle=2 s|r, s\rangle .
$$

We set

$$
F_{r}=\oplus_{s \in \mathbb{Z}} F_{r, s}
$$

A representation of the quantum affine algebra $U_{q}\left(\widehat{s l_{2}}\right)$ is constructed on $F_{r}$ for any $r \in \mathbb{C}$ in [16].

The right Fock module $F_{r, s}^{\dagger}$ and $F_{r}^{\dagger}$ are similarly defined using the vector $\langle r, s|$ satisfying the conditions

$$
\langle r, s| N_{-}=0, \quad\langle r, s| \tilde{a}_{0}=r\langle r, s|, \quad\langle r, s| \tilde{b}_{0}=-2 s\langle r, s|, \quad\langle r, s| \tilde{c}_{0}=2 s\langle r, s| .
$$

Remark 1. We change the definition of $|r, s\rangle$ in [10]. Namely we use

$$
|r, s\rangle=\exp \left(\frac{r}{2(k+2)} Q_{a}+s \frac{Q_{b}+Q_{c}}{2}\right)|0,0\rangle .
$$

Let us introduce field operators which are relevant to our purpose. For $x=a, b, c$ let

$$
\begin{aligned}
& x(L ; M, N \mid z: \alpha)=-\sum_{n \neq 0} \frac{[L n] x_{n}}{[M n][N n]} z^{-n} q^{|n| \alpha}+\frac{L \tilde{x}_{0}}{M N} \log z+\frac{L}{M N} Q_{x}, \\
& x(N \mid z: \alpha)=x(L ; L, N \mid z: \alpha)=-\sum_{n \neq 0} \frac{x_{n}}{[N n]} z^{-n} q^{|n| \alpha}+\frac{\tilde{x}_{0}}{N} \log z+\frac{1}{N} Q_{x} .
\end{aligned}
$$

The normal ordering is defined by specifying $N_{+}, \tilde{a}_{0}, \tilde{b}_{0}, \tilde{c}_{0}$ as annihilation operators and $N_{-}$, $Q_{a}, Q_{b}, Q_{c}$ as creation operators. With this notation let us define the operators

$$
J^{-}(z): F_{r, s} \longrightarrow F_{r, s+1}, \quad \phi_{m}^{(l)}(z): F_{r, s} \longrightarrow F_{r+l, s+l-m}, \quad S(z): F_{r, s} \longrightarrow F_{r-2, s-1},
$$


by

$$
\begin{aligned}
& J^{-}(z)=\frac{1}{\left(q-q^{-1}\right) z}\left(J_{+}^{-}(z)-J_{-}^{-}(z)\right), \\
& J_{\mu}^{-}(z)=: \exp \left(a^{(\mu)}\left(q^{-2} z ;-\frac{k+2}{2}\right)+b\left(2 \mid q^{(\mu-1)(k+2)} z ;-1\right)+c\left(2 \mid q^{(\mu-1)(k+1)-1} z ; 0\right)\right): \\
& a^{(\mu)}\left(q^{-2} z ;-\frac{k+2}{2}\right)=\mu\left\{\left(q-q^{-1}\right) \sum_{n=1}^{\infty} a_{\mu n} z^{-\mu n} q^{\left(2 \mu-\frac{k+2}{2}\right) n}+\tilde{a}_{0} \log q\right\}, \\
& S(z)=\frac{-1}{\left(q-q^{-1}\right) z}\left(S_{+}(z)-S_{-}(z)\right), \\
& S_{\epsilon}(z)=: \exp \left(-a\left(k+2 \mid q^{-2} z ;-\frac{k+2}{2}\right)-b\left(2 \mid q^{-k-2} z ;-1\right)-c\left(2 \mid q^{-k-2+\epsilon} z ; 0\right)\right): \\
& \phi_{l}^{(l)}(z)=: \exp \left(a\left(l ; 2, k+2 \mid q^{k} z ; \frac{k+2}{2}\right)\right):, \\
& \phi_{l-r}^{(l)}(z)=\frac{1}{[r] !} \oint \prod_{j=1}^{r} \frac{d u_{j}}{2 \pi i}\left[\ldots\left[\left[\phi_{l}^{(l)}(z), J^{-}\left(u_{1}\right)\right]_{q^{l}}, J^{-}\left(u_{2}\right)\right]_{q^{l-2}}, \ldots, J^{-}\left(u_{r}\right)\right]_{q^{l-2 r+2}},
\end{aligned}
$$

where

$$
[r] !=[r][r-1] \cdots[1], \quad[X, Y]_{q}=X Y-q Y X,
$$

and the integral in $\phi_{l-r}^{(l)}(z)$ signifies to take the coefficient of $\left(u_{1} \cdots u_{r}\right)^{-1}$.

The operator $J^{-}(z)$ is a generating function of a part of generators of the Drinfeld realization $U_{q}\left(\widehat{s l_{2}}\right)$ at level $k$. While the operators $\phi_{m}^{(l)}(z)$ are conjectured to determine the intertwining operator for $U_{q}\left(\widehat{s l_{2}}\right)$ modules $[10,15]$

$$
\phi^{(l)}(z): W_{r} \longrightarrow W_{r+l} \otimes V_{z}^{(l)}, \quad \phi^{(l)}(z)=\sum_{m=0}^{l} \phi_{m}^{(l)}(z) \otimes v_{m}^{(l)},
$$

where $W_{r}$ is a certain submodule of $F_{r}$ specified as a kernel of a certain operator, called $q$ Wakimoto module $[15,12,13,11,1], V^{(l)}$ is the irreducible representation of $U_{q}\left(s l_{2}\right)$ with spin $l / 2$ and $V_{z}^{(l)}$ is the evaluation representation of $U_{q}\left(\widehat{s l_{2}}\right)$ on $V^{(l)}$.

In this paper we exclusively consider the case $l=1$ and set

$$
\phi_{+}(z)=\phi_{0}^{(1)}, \quad \phi_{-}(z)=\phi_{1}^{(1)}, \quad v_{0}=v_{0}^{(1)}, \quad v_{1}=v_{1}^{(1)} .
$$

The operator $S(z)$ commutes with $U_{q}\left(\widehat{s l_{2}}\right)$ modulo total difference. Here modulo total difference means modulo functions of the form

$$
{ }_{k+2} \partial_{z} f(z):=\frac{f\left(q^{k+2} z\right)-f\left(q^{-(k+2)} z\right)}{\left(q-q^{-1}\right) z} .
$$

Remark 2. The intertwining properties of $\phi^{(l)}(z)$ for $l \in \mathbb{Z}$ are not proved in [10] as pointed out in [15]. However the fact that the matrix elements of compositions of $\phi^{(l)}(z)$ 's and $S(t)$ 's satisfy the $\mathrm{qKZ}$ equation modulo total difference can be proved in a similar way to Proposition 6.1 in [15] using the result of Konno [11] (see (4)).

Let

$$
|m\rangle=|m, 0\rangle \in F_{m, 0}, \quad\langle m|=\langle m, 0| \in F_{m, 0}^{\dagger} .
$$


They become left and right highest weight vectors of $U_{q}\left(\widehat{s l_{2}}\right)$ with the weight $m \Lambda_{1}+(k-m) \Lambda_{0}$ respectively, where $\Lambda_{0}, \Lambda_{1}$ are fundamental weights of $\widehat{s l}_{2}$. Consider

$$
F(t, z)=\left\langle m+n-2 l\left|\phi^{(1)}\left(z_{1}\right) \cdots \phi^{(1)}\left(z_{n}\right) S\left(t_{1}\right) \cdots S\left(t_{l}\right)\right| m\right\rangle
$$

which is a function taking the value in $V^{(1) \otimes n}$. Let

$$
\Delta_{j}=\frac{j(j+2)}{4(k+2)}, \quad s=\frac{1}{2(k+2)} .
$$

Set

$$
\widehat{F}=\left(\prod_{i=1}^{n} z_{i}^{\Delta_{m+n-2 l+1-i}-\Delta_{m+n-2 l-i}}\right) F=\left(\prod_{i=1}^{n} z_{i}^{s\left(m+n-2 l-i+\frac{3}{2}\right)}\right) F,
$$

Let the parameter $p$ be defined from $k$ by

$$
p=q^{2(k+2)} .
$$

We assume $|p|<1$ as before. Then the function $\widehat{F}$ satisfies the following qKZ equation modulo total difference of a function $[15,8,7,10,11]$

$$
T_{j}^{z} \widehat{F}=\widehat{R}_{j, j-1}\left(p z_{j} / z_{j-1}\right) \cdots \widehat{R}_{j, 1}\left(p z_{j} / z_{1}\right) q^{-(m+n / 2-l+1) h_{j}} \widehat{R}_{j, n}\left(z_{j} / z_{n}\right) \cdots \widehat{R}_{j, j+1}\left(z_{j} / z_{j+1}\right) \widehat{F},(4)
$$

where

$$
\begin{aligned}
& \widehat{R}(z)=\rho(z) \tilde{R}(z), \quad \tilde{R}(z)=C^{\otimes 2} R(z) C^{\otimes 2}, \\
& \rho(z)=q^{1 / 2} \frac{\left(z^{-1} ; q^{4}\right)_{\infty}\left(q^{4} z^{-1} ; q^{4}\right)_{\infty}}{\left(q^{2} z^{-1} ; q^{4}\right)_{\infty}^{2}}, \quad(z ; x)_{\infty}=\prod_{i=0}^{\infty}\left(1-x^{i} z\right), \quad C v_{\epsilon}=v_{1-\epsilon} .
\end{aligned}
$$

\section{Integral formulae}

Define the components of $F(t, z)$ by

$$
F(t, z)=\sum_{(\nu) \in\{0,1\}^{n}} F^{(\nu)}(t, z) v_{(\nu)}, \quad v_{(\nu)}=v_{\nu_{1}} \otimes \cdots \otimes v_{\nu_{n}}
$$

where $(\nu)=\left(\nu_{1}, \ldots, \nu_{n}\right)$. By the weight condition $F^{(\nu)}(t, z)=0$ unless the condition

$$
\sharp\left\{i \mid \nu_{i}=0\right\}=l
$$

is satisfied. We assume this condition once for all. Notice that

$$
\begin{aligned}
& \phi_{+}(z)=\frac{1}{\left(q-q^{-1}\right)} \oint \frac{d u}{2 \pi i u}\left[\phi_{-}(z), J_{+}^{-}(u)-J_{-}^{-}(u)\right]_{q}, \\
& S(t)=\frac{-1}{\left(q-q^{-1}\right) t}\left(S_{+}(t)-S_{-}(t)\right) .
\end{aligned}
$$

Let

$$
\left\{i \mid \nu_{i}=0\right\}=\left\{k_{1}<\cdots<k_{l}\right\}
$$


and

$$
\begin{aligned}
F_{(\epsilon)(\mu)}^{(\nu)}(t, z \mid u)= & \langle m+n-2 l| \phi_{-}\left(z_{1}\right) \cdots\left[\phi_{-}\left(z_{k_{1}}\right), J_{\mu_{1}}^{-}\left(u_{1}\right)\right]_{q} \cdots\left[\phi_{-}\left(z_{k_{l}}\right), J_{\mu_{l}}^{-}\left(u_{l}\right)\right]_{q} \cdots \phi_{-}\left(z_{n}\right) \\
& \times S_{\epsilon_{1}}\left(t_{1}\right) \cdots S_{\epsilon_{l}}\left(t_{l}\right)|m\rangle .
\end{aligned}
$$

Then $F^{(\nu)}(t, z)$ can be written as

$$
F^{(\nu)}(t, z)=(-1)^{l}\left(q-q^{-1}\right)^{-2 l} \prod_{a=1}^{l} t_{a}^{-1} \sum_{\epsilon_{i}, \mu_{j}} \prod_{i=1}^{l}\left(\epsilon_{i} \mu_{i}\right) \int_{C^{l}} \prod_{j=1}^{l} \frac{d u_{j}}{2 \pi i u_{j}} F_{(\epsilon)(\mu)}^{(\nu)}(t, z \mid u),
$$

where $C^{l}$ is a suitable deformation of the torus $\mathbb{T}^{l}$ specified as follows.

The contour for the integration variable $u_{i}$ is a simple closed curve rounding the origin in the counterclockwise direction such that $q^{k+3} z_{j}(1 \leq j \leq n), q^{-2} u_{j}(i<j), q^{-\mu_{i}(k+2)} t_{a}(1 \leq a \leq l)$ are inside and $q^{k+1} z_{j}(1 \leq j \leq n), q^{2} u_{j}(j<i)$ are outside.

By the operator product expansions (OPE) of the products of $\phi_{-}(z), J_{\mu}^{-}(u), S_{\epsilon}(t)$ in the appendix, one can compute the function $F_{(\epsilon)(\mu)}^{(\nu)}(t, z \mid u)$ explicitly. In order to write down the formula we need some notation. Set

$$
\xi(z)=\frac{\left(p z^{-1} ; p, q^{4}\right)_{\infty}\left(p q^{4} z^{-1} ; p, q^{4}\right)_{\infty}}{\left(p q^{2} z^{-1} ; p, q^{4}\right)_{\infty}}, \quad(z ; p, q)_{\infty}=\prod_{i=0}^{\infty} \prod_{j=0}^{\infty}\left(1-p^{i} q^{j} z\right) .
$$

Then

$$
F_{(\epsilon)(\mu)}^{(\nu)}(t, z \mid u)=f_{(\mu)}^{(\nu)}(t, z \mid u) \Phi(t, z) G_{(\epsilon)(\mu)}^{(\nu)}(t, z \mid u),
$$

where

$$
\begin{aligned}
f_{(\mu)}^{(\nu)}(t, z \mid u)= & \left(1-q^{2}\right)^{l} q^{\sum_{i=1}^{l}\left(n+m-2 l-k_{i}+i\right) \mu_{i}} \prod_{i=1}^{n}\left(q^{k} z_{i}\right)^{s(m+n-l-i)} \prod_{i<j} \xi\left(z_{i} / z_{j}\right) \\
\times & \prod_{a=1}^{l}\left(q^{-2} t_{a}\right)^{4 s(a-1)-2 m s}, \\
G_{(\epsilon)(\mu)}^{(\nu)}(t, z \mid u)= & \widehat{G}_{(\epsilon)(\mu)}^{(\nu)}(t, z \mid u) \prod_{a<b} \frac{q^{\epsilon_{b}} t_{b}-q^{\epsilon_{a}} t_{a}}{t_{b}-q^{-2} t_{a}}, \\
\widehat{G}_{(\epsilon)(\mu)}^{(\nu)}(t, z \mid u)= & \prod_{i=1}^{l} \frac{u_{i}\left(z_{k_{i}}-q^{\mu_{i}-2-k} u_{i}\right)}{\left(z_{k_{i}}-q^{-1-k} u_{i}\right)\left(u_{i}-q^{k+3} z_{k_{i}}\right)} \prod_{j=1}^{l} \prod_{i<k_{j}} \frac{z_{i}-q^{\mu_{j}-2-k} u_{j}}{z_{i}-q^{-1-k} u_{j}} \\
& \times \prod_{j=1}^{l} \prod_{k_{j}<i} \frac{u_{j}-q^{k+2-\mu_{j}} z_{i}}{u_{j}-q^{k+3} z_{i}} \prod_{i<j} \frac{u_{i}-q^{\mu_{i}-\mu_{j}} u_{j}}{u_{i}-q^{-2} u_{j}} \prod_{i, a} \frac{u_{i}-q^{-\mu_{i}(k+1)-\epsilon_{a}} t_{a}}{u_{i}-q^{-\mu_{i}(k+2)} t_{a}} .
\end{aligned}
$$

Let

$$
G_{(\mu)}^{(\nu)}(t, z)=\sum_{\epsilon_{1}, \ldots, \epsilon_{n}= \pm} \prod_{j=1}^{n} \epsilon_{j} \int_{C^{l}} \prod_{j=1}^{l} \frac{d u_{j}}{2 \pi i u_{j}} G_{(\epsilon)(\mu)}^{(\nu)}(t, z) .
$$

The main theorem in this paper is

Theorem 1. If $(\mu) \neq\left(-^{l}\right)=(-, \ldots,-), G_{(\mu)}^{(\nu)}(t, z)=0$. For $(\mu)=\left(-^{l}\right)$ we have

$$
G_{(-l)}^{(\nu)}(t, z)=q^{-2 l+\frac{1}{2} l(l-1)-\sum_{i=1}^{l} k_{i}}\left(q-q^{-1}\right)^{l} w_{(-\nu)}(t, z),
$$

where $(-\nu)=\left(1-\nu_{1}, \ldots, 1-\nu_{n}\right)$. 
It follows that $F^{(\nu)}(t, z)$ is given by

$$
\begin{aligned}
F^{(\nu)}(t, z)= & (-1)^{l} q^{-(n+m+2-2 l) l+k s n(m+n-l)-\frac{1}{2} k s n(n+1)+4 s l(m-l+1)} \\
& \times \prod_{i=1}^{n} z_{i}^{s(m+n-l-i)} \prod_{i<j} \xi\left(z_{i} / z_{j}\right) \prod_{a=1}^{l} t_{a}^{2 s(2 a-2-m)-1} \Phi(t, z) w_{(-\nu)}(t, z) .
\end{aligned}
$$

\section{Transformation to Tarasov-Varchenko's formulae}

We describe a transformation from $F$, which satisfies (4), to $\Psi$, which satisfies (1). The parameter $\kappa$ is also determined as a function of $l, m, n$.

For a solution $\widehat{G}$ of (4) let

$$
\tilde{G}=\prod_{i=1}^{n} z_{i}^{-s\left(m+\frac{n}{2}-l+1\right)}\left(\left(\prod_{i=1}^{n} z_{i}\right)^{s / 2} \prod_{i<j} \xi\left(z_{i} / z_{j}\right)\right)^{-1} C^{\otimes n} \widehat{G}
$$

One can easily verify that $\tilde{G}$ satisfies (1) with $\kappa=q^{2 l-2-n-2 m}$ using

$$
\frac{\xi(p z)}{\xi(z)}=\frac{\left(z^{-1} ; q^{4}\right)_{\infty}\left(q^{4} z^{-1} ; q^{4}\right)_{\infty}}{\left(q^{2} z^{-1} ; q^{4}\right)_{\infty}^{2}}
$$

Let $\widehat{F}$ be defined by (3) and $\tilde{F}$ by (5). Then

$$
\begin{aligned}
\tilde{F}= & (-1)^{l} q^{-(n+m+2-2 l) l+k s n(m+n-l)-\frac{1}{2} k s n(n+1)+4 s l(m-l+1)} \\
& \times \prod_{i=1}^{n} z_{i}^{s(m+n-2 l-i)} \prod_{a=1}^{l} t_{a}^{2 s(2 a-2-m)-1} \Phi(t, z) \sum w_{(\nu)}(t, z) \otimes v_{(\nu)} .
\end{aligned}
$$

For $W \in \mathcal{F}_{\text {ell }}$ let

$$
\tilde{W}=W\left(\prod_{i=1}^{n} z_{i}^{s(m+n-2 l-i)} \prod_{a=1}^{l} t_{a}^{2 s(2 a-2-m)}\right)^{-1} .
$$

Then the condition (iii) for $W$ is equivalent to the following conditions,

$$
T_{a}^{t} \tilde{W} / \tilde{W}=1, \quad T_{j}^{z} \tilde{W} / \tilde{W}=q^{l-m-n+j} .
$$

To sum up we have

Proposition 1. For any $W \in \mathcal{F}_{\text {ell }}$

$$
\tilde{\Psi}_{W}=\int_{\tilde{\mathbb{T}}^{l}} \prod_{a=1}^{l} \frac{d t_{a}}{2 \pi i} \tilde{F}(t, z) \tilde{W}(t, z)
$$

is a solution of the $q K Z$ equation (1), where $\tilde{F}$ is defined by (5) with $\widehat{F}$ and $F$ being given in (3) and (2) and $\tilde{W}$ is defined by (6). 


\section{Proof of Theorem}

Let $A^{ \pm}=\left\{j \mid \mu_{j}= \pm\right\}$. Suppose that the number of elements in $A^{ \pm}$is $r^{ \pm}$and write $A^{ \pm}=\left\{l_{1}^{ \pm}<\right.$ $\cdots<l_{r^{ \pm}}^{ \pm}$. Set $A=A^{-}, r=r^{-}$and $l_{i}=l_{i}^{-}$for simplicity. Let

$$
I_{(\epsilon)(\mu)}^{(\nu)}(t, z)=\int_{C^{l}} \prod_{j=1}^{l} \frac{d u_{j}}{2 \pi i u_{j}} \widehat{G}_{(\epsilon)(\mu)}^{(\nu)}(t, z \mid u) .
$$

Lemma 1. We have

$$
\begin{aligned}
I_{(\epsilon)(\mu)}^{(\nu)}(t, z)= & \left(-q^{-2}\right)^{r}\left(q-q^{-1}\right)^{r} \sum_{\substack{a_{1}, \ldots, a_{r}=1 \\
a_{i} \neq a_{j}(i \neq j)}}^{l} \prod_{i=1}^{r} \delta_{\epsilon_{a_{i}},+} \prod_{i=1}^{r} \frac{t_{a_{i}}}{z_{k_{l_{i}}}-q t_{a_{i}}} \prod_{j=1}^{r} \prod_{i<k_{l_{j}}} \frac{z_{i}-q^{-1} t_{a_{j}}}{z_{i}-q t_{a_{j}}} \\
& \times \prod_{i=1}^{r} \prod_{a \neq a_{i}, \ldots, a_{r}} \frac{t_{a_{i}}-q^{-1-\epsilon_{a}} t_{a}}{t_{a_{i}}-t_{a}} .
\end{aligned}
$$

Proof. We first integrate in the variables $u_{j}, j \in A^{+}$in the order $u_{l_{1}^{+}}, \ldots, u_{l_{r^{+}}}$. Let us consider the integration in $u_{l_{1}^{+}}$. We denote the integration contour in $u_{i}$ by $C_{i}$. The only singularity of the integrand outside $C_{l_{1}^{+}}$is $\infty$. Thus the integral is calculated by taking residue at $\infty$. Since the integrand is of the form

$$
\frac{d u_{l_{1}^{+}}}{u_{l_{1}^{+}}} H\left(u_{l_{1}^{+}}\right)
$$

where $H(u)$ is holomorphic at $\infty$. Then

$$
\int_{C_{l_{1}^{+}}} \frac{d u}{2 \pi i u} H(u)=-\operatorname{Res}_{u=\infty} \frac{d u}{u} H(u)=\lim _{u \longrightarrow \infty} H(u) .
$$

In this way the integral in $u_{l_{1}^{+}}$is calculated. After this integration the integrand as a function of $u_{l_{2}^{+}}$has a similar structure. Therefore the integration with respect to $u_{l_{2}^{+}}$is carried out in a similar way and so on. Finally we get

$$
\begin{aligned}
& I_{(\epsilon)(\mu)}^{(\nu)}(t, z)=(-1)^{r^{+}} \operatorname{Res}_{\substack{u_{l^{+}}=\infty \\
r^{+}}} \cdots \operatorname{Res}_{u_{l_{1}^{+}}=\infty} \widehat{G}_{(\epsilon)(\mu)}^{(\nu)}(t, z \mid u) \\
& =\int_{C^{n-r}+} \prod_{j \in A} \frac{d u_{j}}{2 \pi i u_{j}} \prod_{i \in A} \frac{-q^{-3-k} u_{i}}{z_{k_{i}}-q^{-1-k} u_{i}} \prod_{\substack{i<k_{j} \\
j \in A}} \frac{z_{i}-q^{-3-k} u_{j}}{z_{i}-q^{-1-k} u_{j}} \prod_{\substack{i<j \\
i, j \in A}} \frac{u_{i}-u_{j}}{u_{i}-q^{-2} u_{j}} \\
& \times \prod_{\substack{i \in A \\
a}} \frac{u_{i}-q^{k+1-\epsilon_{a}} t_{a}}{u_{i}-q^{k+2} t_{a}}
\end{aligned}
$$

Here $C^{n-r^{+}}$is specified by similar conditions to $C^{l}$, where $u_{l_{i}^{+}} 1 \leq i \leq r^{+}$are omitted. We denote the right hand side of this equation other than $\int_{C^{n-r}+} \prod_{j} \frac{d u_{j}}{2 \pi i u_{j}}$ by $I_{(\epsilon)(\mu)}^{(\nu)+}(t, z)$.

Next we integrate with respect to the remaining variables $u_{j}, j \in A$ in the order $u_{l_{r}}, \ldots, u_{l_{1}}$. Let us consider the integration with respect to $u_{l_{r}}$. The poles of the integrand inside $C_{l_{r}}$ is 
$q^{k+2} t_{a}, a=1, \ldots, l$. Thus we have

$$
\begin{aligned}
& \int_{C_{l_{r}}} \frac{d u_{l_{r}}}{2 \pi i u_{l_{r}}} I_{(\epsilon)(\mu)}^{(\nu)+}(t, z)=\sum_{a_{r}=1}^{l} \operatorname{Res}_{u_{l_{r}}=q^{k+2} t_{a_{r}}} I_{(\epsilon)(\mu)}^{(\nu)+}(t, z) \\
& =\sum_{a_{r}=1}^{l} \prod_{\substack{i \in A \\
i \neq l_{r}}} \frac{-q^{-3-k} u_{i}}{z_{k_{i}}-q^{-1-k} u_{i}} \prod_{\substack{i<k_{j} \\
j \in A \backslash\left\{l_{r}\right\}}} \frac{z_{i}-q^{-3-k} u_{j}}{z_{i}-q^{-1-k} u_{j}} \prod_{\substack{i<j<l_{r} \\
i, j \in A}} \frac{u_{i}-u_{j}}{u_{i}-q^{-2} u_{j}} \prod_{\substack{i \in A \backslash\left\{l_{r}\right\} \\
a}} \frac{u_{i}-q^{k+1-\epsilon_{a}} t_{a}}{u_{i}-q^{k+2} t_{a}} \\
& \times \frac{-q^{-1} t_{a_{r}}}{z_{k_{l_{r}}}-q t_{a_{r}}} \prod_{i<k_{l_{r}}} \frac{z_{i}-q^{-1} t_{a_{r}}}{z_{i}-q t_{a_{r}}} \prod_{\substack{i<k_{l_{r}} \\
i \in A}} \frac{u_{i}-q^{k+2} t_{a_{r}}}{u_{i}-q^{k} t_{a_{r}}} \prod_{a \neq a_{r}} \frac{t_{a_{r}}-q^{-1-\epsilon_{a}} t_{a}}{t_{a_{r}}-t_{a}}\left(1-q^{-1-\epsilon_{a}}\right) \\
& =\left(1-q^{-2}\right) \sum_{a_{r}=1}^{l} \delta_{\epsilon_{a_{r}},+} \prod_{\substack{i \in A \\
i \neq l_{r}}} \frac{-q^{-3-k} u_{i}}{z_{k_{i}}-q^{-1-k} u_{i}} \prod_{\substack{i<k_{j} \\
j \in A \backslash\left\{l_{r}\right\}}} \frac{z_{i}-q^{-3-k} u_{j}}{z_{i}-q^{-1-k} u_{j}} \prod_{\substack{i<j<l_{r} \\
i, j \in A}} \frac{u_{i}-u_{j}}{u_{i}-q^{-2} u_{j}} \\
& \times\left(\prod_{\substack{i \in A \backslash\left\{l_{r}\right\} \\
a \neq a_{r}}} \frac{u_{i}-q^{k+1-\epsilon_{a}} t_{a}}{u_{i}-q^{k+2} t_{a}}\right) \frac{-q^{-1} t_{a_{r}}}{z_{k_{l_{r}}}-q t_{a_{r}}} \prod_{i<k_{l_{r}}} \frac{z_{i}-q^{-1} t_{a_{r}}}{z_{i}-q t_{a_{r}}} \prod_{a \neq a_{r}} \frac{t_{a_{r}}-q^{-1-\epsilon_{a}} t_{a}}{t_{a_{r}}-t_{a}} .
\end{aligned}
$$

The last expression as a function of $u_{l_{r-1}}$ has a similar form to $I_{(\epsilon)(\mu)}^{(\nu)+}(t, z)$ as a function of $u_{l_{r}}$. Namely the poles inside $C_{l_{r-1}}$ are $q^{k+2} t_{a}, a \neq a_{r}$. Thus the integral in $u_{l_{r-1}}$ is the sum of residues at $q^{k+2} t_{a}, a \neq a_{r}$ and so on. Finally we get

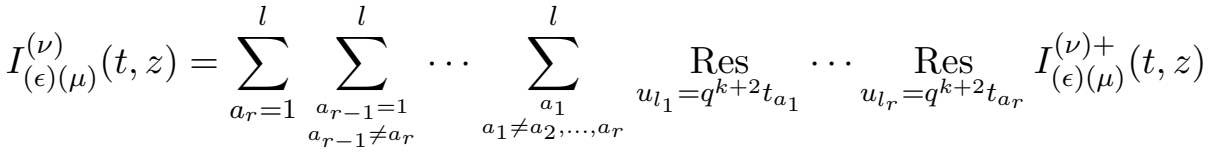

$$
\begin{aligned}
& =\left(-q^{-2}\right)^{r}\left(q-q^{-1}\right)^{r} \sum_{\substack{a_{1}, \ldots, a_{r}=1 \\
a_{i} \neq a_{j}(i \neq j)}}^{l} \prod_{i=1}^{r} \delta_{\epsilon_{a_{i}},+} \prod_{i=1}^{r} \frac{t_{a_{i}}}{z_{k_{l_{i}}}-q t_{a_{i}}} \prod_{j=1}^{r} \prod_{i<k_{l_{j}}} \frac{z_{i}-q^{-1} t_{a_{j}}}{z_{i}-q t_{a_{j}}} \\
& \times \prod_{i=1}^{r} \prod_{a \neq a_{i}, \ldots, a_{r}} \frac{t_{a_{i}}-q^{-1-\epsilon_{a}} t_{a}}{t_{a_{i}}-t_{a}} .
\end{aligned}
$$

Thus the lemma is proved.

Recall that

$$
G_{(\epsilon)(\mu)}^{(\nu)}(t, z)=I_{(\epsilon)(\mu)}^{(\nu)}(t, z) \prod_{a<b} \frac{q^{\epsilon_{b}} t_{b}-q^{\epsilon_{a}} t_{a}}{t_{b}-q^{-2} t_{a}} .
$$

For a set $\left\{a_{1}, \ldots, a_{r}\right\}$ let $\left\{b_{1}, \ldots, b_{r^{+}}\right\}$be defined by

$$
\{1, \ldots, l\}=\left\{a_{i}\right\} \cup\left\{b_{i}\right\} .
$$

Then

$$
\begin{aligned}
& \left(-q^{2}\right)^{r}\left(q-q^{-1}\right)^{-r} G_{(\mu)}^{(\nu)}(t, z) \\
& =\sum_{\substack{a_{1}, \ldots, a_{r}=1 \\
a_{i} \neq a_{j}(i \neq j)}}^{l} \prod_{i=1}^{r} \frac{t_{a_{i}}}{z_{k_{l_{i}}}-q t_{a_{i}}} \prod_{i=1}^{r} \prod_{j<k_{l_{i}}} \frac{z_{j}-q^{-1} t_{a_{i}}}{z_{j}-q t_{a_{i}}} \prod_{i>j} \frac{t_{a_{i}}-q^{-2} t_{a_{j}}}{t_{a_{i}}-t_{a_{j}}} \prod_{i=1}^{r} \prod_{j=1}^{r^{+}} \frac{1}{t_{a_{i}}-t_{b_{j}}} \prod_{a<b} \frac{1}{t_{b}-q^{-2} t_{a}} \\
& \quad \times \prod_{i=1}^{r} \delta_{\epsilon_{a_{i}},+} \sum_{\epsilon_{b_{1}, \ldots, \epsilon_{b_{r^{+}}}}= \pm} \prod_{j=1}^{r^{+}} \epsilon_{b_{j}} \prod_{i=1}^{r} \prod_{j=1}^{r^{+}}\left(t_{a_{i}}-q^{-1-\epsilon_{b_{j}}} t_{b_{j}}\right) \prod_{a<b}\left(q^{\epsilon_{b}} t_{b}-q^{\epsilon_{a}} t_{a}\right) .
\end{aligned}
$$


Let us calculate the sum in $\left\{\epsilon_{b_{i}}\right\}$ assuming $\epsilon_{a_{i}}=+$. Using

$$
\left(t_{a}-q^{-1-\epsilon_{b}} t_{b}\right)\left(t_{a}-q^{-1+\epsilon_{b}} t_{b}\right)=\left(t_{a}-t_{b}\right)\left(t_{a}-q^{-2} t_{b}\right),
$$

and

$$
\begin{aligned}
\prod_{a<b}\left(q^{\epsilon_{b}} t_{b}-q^{\epsilon_{a}} t_{a}\right)= & \prod_{\substack{i, j \\
a_{i}>a_{j}}} q\left(t_{a_{i}}-t_{a_{j}}\right) \prod_{\substack{i, j \\
b_{i}>b_{j}}}\left(q^{\epsilon_{b_{i}}} t_{b_{i}}-q^{\epsilon_{b_{j}}} t_{b_{j}}\right) \\
& \times \prod_{\substack{i, j \\
a_{i}>b_{j}}}\left(q t_{a_{i}}-q^{\epsilon_{b_{j}}} t_{b_{j}}\right) \prod_{\substack{i, j \\
b_{j}>a_{i}}}\left(q^{\epsilon_{b_{j}}} t_{b_{j}}-q t_{a_{i}}\right),
\end{aligned}
$$

we have

$$
\begin{aligned}
& \sum_{\epsilon_{b_{1}, \ldots, \epsilon_{b^{+}}}= \pm} \prod_{j=1}^{r^{+}} \epsilon_{b_{j}} \prod_{i=1}^{r} \prod_{j=1}^{r^{+}}\left(t_{a_{i}}-q^{-1-\epsilon_{b_{j}}} t_{b_{j}}\right) \prod_{a<b}\left(q^{\epsilon_{b}} t_{b}-q^{\epsilon_{a}} t_{a}\right) \\
& =(-1)^{l r-\frac{1}{2} r(r-1)-\sum_{i=1}^{r} a_{i}} q^{r r^{+}+\frac{1}{2} r(r-1)} \prod_{i=1}^{r} \prod_{j=1}^{r^{+}}\left(t_{a_{i}}-t_{b_{j}}\right)\left(t_{a_{i}}-q^{-2} t_{b_{j}}\right) \prod_{\substack{i, j \\
a_{i}>a_{j}}}\left(t_{a_{i}}-t_{a_{j}}\right) \\
& \quad \times \sum_{\epsilon_{b_{1}, \ldots, \epsilon_{r^{+}}}= \pm} \prod_{j=1}^{r^{+}} \epsilon_{b_{j}} \prod_{\substack{i, j \\
b_{i}>b_{j}}}\left(q^{\epsilon_{b_{i}}} t_{b_{i}}-q^{\epsilon_{b_{j}}} t_{b_{j}}\right) .
\end{aligned}
$$

Lemma 2. For $N \geq 1$ we have

$$
\sum_{\epsilon_{1}, \ldots, \epsilon_{N}= \pm} \prod_{j=1}^{N} \epsilon_{j} \prod_{i>j}\left(q^{\epsilon_{i}} t_{i}-q^{\epsilon_{j}} t_{j}\right)=0
$$

Proof. Let

$$
\mathbf{a}_{i}(\epsilon)={ }^{t}\left(1, q^{\epsilon} t_{i},\left(q^{\epsilon} t_{i}\right)^{2}, \ldots,\left(q^{\epsilon} t_{i}\right)^{N-1}\right) .
$$

Then the left hand side of (8) is equal to

$$
\sum_{\epsilon_{1}, \ldots, \epsilon_{N}= \pm} \prod_{j=1}^{N} \epsilon_{j} \operatorname{det}\left(\mathbf{a}_{1}\left(\epsilon_{1}\right), \ldots, \mathbf{a}_{N}\left(\epsilon_{N}\right)\right)=\operatorname{det}\left(\sum_{\epsilon_{1}} \epsilon_{1} \mathbf{a}_{1}\left(\epsilon_{1}\right), \ldots, \sum_{\epsilon_{N}} \epsilon_{N} \mathbf{a}_{N}\left(\epsilon_{N}\right)\right) .
$$

Since

$$
\sum_{\epsilon} \epsilon \mathbf{a}_{i}(\epsilon)={ }^{t}\left(0,\left(q-q^{-1}\right) t_{i}, \ldots,\left(q^{N-1}-q^{-(N-1)}\right) t_{i}^{N-1}\right),
$$

the right hand side of (9) is zero.

By this lemma the right hand side of (7) becomes zero if $r^{+}>0$. Consequently $G_{(\mu)}^{(\nu)}=0$ for $r^{+}>0$. Suppose that $r^{+}=0$. In this case $r=l, l_{i}=i(1 \leq i \leq l)$ and

$$
\begin{aligned}
& \left(-q^{2}\right)^{l}\left(q-q^{-1}\right)^{-l} G_{(-l)}^{(\nu)}(t, z) \\
& \quad=\prod_{a<b} \frac{q\left(t_{b}-t_{a}\right)}{t_{b}-q^{-2} t_{a}} \sum_{\substack{a_{1}, \ldots, a_{l}=1 \\
a_{i} \neq a_{j}(i \neq j)}}^{l} \prod_{i=1}^{l}\left(\frac{t_{a_{i}}}{z_{k_{i}}-q t_{a_{i}}} \prod_{j<k_{i}} \frac{z_{j}-q^{-1} t_{a_{i}}}{z_{j}-q t_{a_{i}}} \prod_{i>j} \frac{t_{a_{i}}-q^{-2} t_{a_{j}}}{t_{a_{i}}-t_{a_{j}}}\right) .
\end{aligned}
$$

The theorem easily follows from this. 


\section{Concluding remarks}

In this paper we study the solutions of the $\mathrm{qKZ}$ equation taking the value in the tensor product of the two dimensional evaluation representation of $U_{q}\left(\widehat{s l}_{2}\right)$. The integral formulae are derived for the highest to highest matrix elements for certain intertwining operators by using free field realizations. The integrals with respect to $u$ variables corresponding to the operator $J^{-}(u)$ are calculated and the sum arising from the expression of $J^{-}(u)$ and the screening operator $S(t)$ is calculated. The formulae thus obtained coincide with those of Tarasov and Varchenko. The calculations in this paper can be extended to the case where the vector space $V^{(1) \otimes n}$ is replaced by a tensor product of more general representations. It is an interesting problem to perform similar calculations for other quantum affine algebras [2] and the elliptic algebras [13].

In Tarasov-Varchenko's theory solutions of a qKZ equation are parametrized by elements of the elliptic hypergeometric space $\mathcal{F}_{\text {ell }}$ while the matrix elements are specified by intertwiners. It is an interesting problem to establish a correspondence between intertwining operators and elements of $\mathcal{F}_{\text {ell }}$. With the results of the present paper one can begin to study this problem. Study in this direction will provide a new insight on the space of local fields and correlation functions of integrable field theories and solvable lattice models. The corresponding problem in CFT is studied in [5].

\section{Appendix. List of OPE's}

Here we list OPE's which are necessary in this paper. Almost all of them are taken from the paper [10]. Let

$$
\begin{aligned}
& C(z)=\frac{\left(q^{-2} z ; p\right)_{\infty}}{\left(q^{2} z ; p\right)_{\infty}}, \quad(z)_{\infty}=(z ; p)_{\infty} . \\
& S_{\epsilon_{1}}\left(t_{1}\right) S_{\epsilon_{2}}\left(t_{2}\right)=\left(q^{-2} t_{1}\right)^{4 s} q^{\epsilon_{1}} \frac{t_{1}-q^{\epsilon_{2}-\epsilon_{1}} t_{2}}{t_{1}-q^{-2} t_{2}} C\left(t_{2} / t_{1}\right): S_{\epsilon_{1}}\left(t_{1}\right) S_{\epsilon_{2}}\left(t_{2}\right):, \quad\left|q^{-2} t_{2}\right|<\left|t_{1}\right|, \\
& \phi_{-}(z) S_{\epsilon}(t)=\left(q^{k} z\right)^{-s} \frac{(q t / z)_{\infty}}{\left(q^{-1} t / z\right)_{\infty}}: \phi_{-}(z) S_{\epsilon}(t):, \quad\left|q^{-1} t\right|<|z|, \\
& J_{\mu}^{-}(u) S_{\epsilon}(t)=q^{-\mu} \frac{u-q^{-\mu(k+1)-\epsilon} t}{u-q^{-\mu(k+2)} t}: J_{\mu}^{-}(u) S_{\epsilon}(t):, \quad\left|q^{-(k+2)} t\right|<|u|, \\
& \phi_{-}(z) J_{\mu}^{-}(u)=\frac{z-q^{\mu-2-k} u}{z-q^{-1-k} u}: \phi_{-}(z) J_{\mu}^{-}(u):, \quad|u|<\left|q^{k+3} z\right| \quad \text { for } \quad \mu=-, \\
& J_{\mu}^{-}(u) \phi_{-}(z)=q^{\mu} \frac{u-q^{k+2-\mu} z}{u-q^{k+3} z}: \phi_{-}(z) J_{\mu}^{-}(u):, \quad\left|q^{k+1} z\right|<|u| \quad \text { for } \quad \mu=+, \\
& {\left[\phi_{-}(z), J_{\mu}^{-}(u)\right]_{q}=\frac{\left(1-q^{2}\right) u\left(z-q^{\mu-2-k} u\right)}{\left(z-q^{-1-k} u\right)\left(u-q^{k+3} z\right)}: \phi_{-}(z) J_{\mu}^{-}(u):,} \\
& J_{\mu_{1}}^{-}\left(u_{1}\right) J_{\mu_{2}}^{-}\left(u_{2}\right)=q^{-\mu_{1}} \frac{u_{1}-q^{\mu_{1}-\mu_{2}} u_{2}}{u_{1}-q^{-2} u_{2}}: J_{\mu_{1}}^{-}\left(u_{1}\right) J_{\mu_{2}}^{-}\left(u_{2}\right):, \quad\left|q^{-2} u_{2}\right|<\left|u_{1}\right|, \\
& \phi_{-}\left(z_{1}\right) \phi_{-}\left(z_{2}\right)=\left(q^{k} z_{1}\right)^{s} \xi\left(z_{1} / z_{2}\right): \phi_{-}\left(z_{1}\right) \phi_{-}\left(z_{2}\right):, \quad\left|p z_{2}\right|<\left|z_{1}\right| .
\end{aligned}
$$

Note 1. After completing the paper we were informed that H. Awata, S. Odake and J. Shiraishi obtained a similar result to this paper. The result is reviewed in Shiraishi's PhD thesis [17] in which one statement is a conjecture. However the complete version containing all proofs for all statements had not been published after all. We would like to thank J. Shiraishi for the kind correspondence. 


\section{Acknowledgements}

We would like to thank Hitoshi Konno and Yasuhiko Yamada for valuable discussions and comments. We are also grateful to Atsushi Matsuo for useful comments on the manuscript.

\section{References}

[1] Abada A., Bougourzi A.H., El Gradechi M.A., Deformation of the Wakimoto construction, Modern Phys. Lett. A 8 (1993), 715-724, hep-th/9209009.

[2] Awata H., Odake S., Shiraishi J., Free boson realization of $U_{q}\left(\widehat{s l}_{N}\right)$, Comm. Math. Phys. 162 (1994), 61-83, hep-th/9305146.

[3] Awata H., Tsuchiya A., Yamada Y., Integral formulas for the WZNW correlation functions, Nuclear Phys. B 365 (1991), 680-696.

[4] Bougourzi A.H., Weston R.A., Matrix elements of $U_{q}\left(s u(2)_{k}\right)$ vertex operators via bosonization, Internat. J. Modern Phys. A 9 (1994), 4431-4447, hep-th/9305127.

[5] Felder G., BRST approach to minimal models, Nuclear Phys. B 317 (1989), 215-236.

[6] Frenkel I.B., Jing N.H., Vertex representations of quantum affine algebras, Proc. Nat. Acad. Sci. U.S.A. 85 (1988), 9373-9377.

[7] Frenkel I.B., Reshetikhin N.Yu., Quantum affine algebras and holonomic difference equations, Comm. Math. Phys. 146 (1992), 1-60.

[8] Idzumi M., Tokihiro T., Iohara K., Jimbo M., Miwa T., Nakashima T., Quantum affine symmetry in vertex models, Internat. J. Modern Phys. A 8 (1993), 1479-1511, hep-th/9208066.

[9] Jimbo M., Miwa T., Algebraic analysis of solvable lattice models, CBMS Regional Conference Series in Mathematics, Vol. 85, American Math. Soc., Providence, RI, 1995.

[10] Kato A., Quano Y.-H., Shiraishi J., Free boson representation of $q$-vertex operators and their correlation functions, Comm. Math. Phys. 157 (1993), 119-137, hep-th/9209015.

[11] Konno H., BRST cohomology in quantum affine algebra $U_{q}\left(\widehat{s l}_{2}\right)$, Modern Phys. Lett. A 9 (1994), 1253-1265, hep-th/9310108.

[12] Konno H., Free-field representation of the quantum affine algebra $U_{q}\left(\widehat{s l}_{2}\right)$ and form factors in the higher-spin XXZ model, Nuclear Phys. B 432 (1994), 457-486, hep-th/9407122.

[13] Konno H., An elliptic algebra $U_{q, p}\left(\widehat{s l}_{2}\right)$ and the fusion RSOS model, Comm. Math. Phys. 195 (1998), 373-403, q-alg/9709013.

[14] Matsuo A., Quantum algebra structure of certain Jackson integrals, Comm. Math. Phys. 157 (1993), $479-$ 498.

[15] Matsuo A., A q-deformation of Wakimoto modules, primary fields and screening operators, Comm. Math. Phys. 160 (1994), 33-48, hep-th/9212040.

[16] Shiraishi J., Free boson representation of quantum affine algebra, Phys. Lett. A 171 (1992), 243-248.

[17] Shiraishi J., Free boson realization of quantum affine algebras, PhD thesis, University of Tokyo, 1995.

[18] Schechtman V.V., Varchenko A.N., Integral representations of $N$-point conformal correlators in the WZW model, Max-Planck-Institut fur Mathematik, Preprint MPI/89-51, 1989.

[19] Schechtman V.V., Varchenko A.N., Hypergeometric solutions of Knizhnik-Zamolodchikov equations, Lett. Math. Phys. 20 (1990), 279-283.

[20] Tarasov V., Hypergeometric solutions of the qKZ equation at level zero, Czechoslovak J. Phys. 50 (2000), 193-200.

[21] Varchenko A.N., Tarasov V.O., Jackson integral representations of solutions of the quantized KnizhnikZamolodchikov equation, St. Petersburg Math. J. 6 (1995), 275-313, hep-th/9311040.

[22] Tarasov V., Varchenko A., Geometry of $q$-hypergeometric functions, quantum affine algebras and elliptic quantum groups, Astérisque 246 (1997), 1-135.

[23] Varchenko A., Quantized Knizhnik-Zamolodchikov equations, quantum Yang-Baxter equation, and difference equations for $q$-hypergeometric functions, Comm. Math. Phys. 162 (1994), 499-528. 